\title{
FUNDAMENTOS DE DISEÑO DE UN TALLER DE POSGRADO SOBRE “INTERVENCIONES INTERDISCIPLINARIAS”
}

\author{
Patricia Silvana San Martín (CIFASIS -CONICET-UNR-AMU)* \\ sanmartin@cifasis-conicet.gov.ar
}

Recibido: 07/08/2012 Aceptado: 16/10/2012

\begin{abstract}
Resumen
El artículo fundamenta una experiencia de diseño y puesta en obra de un taller físico-virtual denominado "Intervenciones Interdisciplinarias", atendiendo a diversas problemáticas sociales complejas que se presentan en la Argentina. El mismo, es parte de un proyecto de carrera de especialización (nivel posgrado), propuesto por una universidad ubicada en la región del noroeste del país, que aborda problemáticas subjetivas del contexto jurídico. El texto expone el diseño del taller y su puesta en obra a partir del marco téorico-metodológico del Dispositivo Hipermedial Dinámico. A su vez, se describen y documentan aspectos inherentes al desarrollo del taller, analizando algunos resultados provisorios alcanzados por la actual cohorte, integrada por profesionales del Derecho, Psicología y Trabajo Social, entre otros. Finalmente, las conclusiones ponen a consideración el significativo aporte que la participación en redes sociotécnicas colaborativas, podría brindar hacia la construcción de la disponibilidad interdisciplinar en función de mejorar las prácticas físico-virtuales profesionales y/o de formación superior. En este sentido, se enfatiza la necesidad de construcción de Dispositivos Hipermediales Dinámicos, fundamentados en la ética de la SubjetividadResponsabilidad, los Derechos Humanos y la adhesión a las perspectivas internacionales de Acceso Abierto y Código Abierto.
\end{abstract}

\section{Palabras Clave}

Educación Superior - Interdisciplinariedad - Dispositivo Hipermedial Dinámico - Taller - Contexto Jurídico.

* Doctora en Humanidades y Artes. Universidad Nacional de Rosario. Profesora Nacional de Música. Universidad Nacional de Rosario. Investigadora Adjunta del CONICET. 


\begin{abstract}
The paper presents a design experience and application of a physical-virtual workshop entitled "Interdisciplinary Intervention", in response to various complex social problems that arise today in Argentina. This workshop is part of a project of graduate career specialization, accredited by a university in the northwestern region of the country, which addresses issues of subjective legal context. Its design was based from theoretical and methodological framework of Dynamic Hypermedia Device. The text also describes aspects about the development of the workshop, analyzing some interim results achieved by the present cohort, comprised of legal professionals, psychology and social work, among others. Finally, the findings put to consideration the significant contribution that participation in collaborative sociotechnical networks could provide towards building interdisciplinary availability in terms of improving the physicalvirtual professional practices. In this regard, we emphasize the need to build Dynamic Hypermedia Devices, based on the ethics of Subjectivity-Responsibility, Human Rights and adherence to international perspectives on Open Access and Open Source.
\end{abstract}

\title{
Key words
}

Higher Education - Interdisciplinarity - Dynamic Hypermedia Device - Workshop - Legal Context.

\section{Introducción}

El artículo introduce los fundamentos que sustentaron el diseño y puesta en obra de un taller denominado "Intervenciones Interdisciplinarias" que forma parte de un proyecto de carrera de especialización de posgrado universitario donde se aborda el estudio de problemáticas subjetivas del contexto jurídico presentes en la República Argentina. La finalidad del escrito es exponer dicha experiencia a modo de caso propositivo, poniendo a consideración perspectivas y procesos que podrían habilitar en el actual contexto físico-virtual, la construcción de la disponibilidad interdisciplinar y sus efectos tanto en el marco de un trayecto de formación de posgrado como en diversas prácticas profesionales.

En relación al proyecto de carrera, la misma fue propuesta por una universidad situada en la capital de la provincia de Salta (región del noroeste argentino- NOA), se encuentra acreditada por la Comisión Nacional de Evaluación y Acreditación Universitaria (CONEAU) y tiene por destinatarios a profesionales provenientes de distintas disciplinas de las Ciencias Sociales y Humanas. Actualmente, se está desarrollando la primera cohorte (2011-2012) con modalidad de cursado presencial integrando Tecnologías de la Información y Comunicación (TIC).

El objetivo general de la especialización se centra en formar especialistas de posgrado que, siendo parte de un equipo multidisciplinario, puedan construir marcos conceptuales y metodológicos comunes fundamentados en un enfoque sistémico complejo, sustentados en prácticas de investigación interdisciplinaria 
y propuestas de acción, asumiendo el compromiso social y/o generación de políticas públicas de prevención desde posiciones éticas con la Subjetividad y los Derechos Humanos.

La necesidad de esta especialización surgió al observar que en Argentina, los campos disciplinares intervinientes en los casos judicializados, se encuentran en las instituciones de referencia fuertemente parcelados, poniendo en evidencia variadas problemáticas en las prácticas profesionales (Degano, 2005). En este sentido, se presenta preocupante la ausencia de sistematicidad y cohesión del trabajo interdisciplinario en el marco de las actuales Juntas Interdisciplinarias, la baja integración de recursos TIC para el trabajo colaborativo de los equipos intervinientes en relación a las causas en trámite, como así también la constatación de debilidades en la formación profesional con respecto al abordaje de casos provenientes de poblaciones diversas (migraciones internas y externas, presencia de pueblos originarios, mestizajes, entre otros), en condiciones de riesgo social que dan cuenta de distancias culturales profundas entre los actores involucrados.

Ante este conjunto de problemas, el enfoque de Rolando García sobre la metodología de trabajo interdisciplinario en el marco de los sistemas complejos, se evalúa como un aporte significativo y consistente, ya que otorga validados antecedentes y fundamentados lineamientos de aplicabilidad para sustentar tanto una perspectiva de formación en el nivel de posgrado como el desarrollo de prácticas profesionales en contextos complejos. Siguiendo a García (2007):

Un sistema complejo es un sistema en el cual los procesos que determinan su funcionamiento son el resultado de la confluencia de múltiples factores que interactúan de tal manera que el sistema no es descomponible sino sólo semi-descomponible. Por lo tanto, ningún sistema complejo puede ser descrito por la simple adición de estudios independientes sobre cada uno de los componentes. (p.182)

En relación a la elaboración del diseño curricular de la especialización y su posterior implementación, se decidió articular en dicho proyecto de carrera instancias integradoras y participativas que reconozcan la heterogeneidad disciplinar del grupo destinatario (Derecho, Psicología, Trabajo Social, Psiquiatría, Educación, etc.); la parcelación y fragmentación de las prácticas institucionales jurídicas donde la mayoría de las/los cursantes trabajan; las representaciones a nivel de pre-juicios sobre el otro que cada uno porta en su experiencia profesional y, la complejidad de los casos en trámite que se presentan en la región del NOA.

Estos múltiples factores -que no operan por simple adición- dan clara cuenta por un lado de la complejidad sistémica que se debía abordar a nivel curricular y por otro de la necesidad de construcción por parte de las/los destinatarios de un marco teórico común, sustentado en el diálogo interdisciplinario. En este 
sentido, el taller físico-virtual "Intervenciones Interdisciplinarias", situado en el trayecto medio de la carrera, se consideró clave para promover el diálogo sistemático sobre las problemáticas subjetivas del contexto jurídico conceptualizándolo como un espacio participativo privilegiado que podía dar comienzo al desarrollo de herramientas analíticas adecuadas a los sistemas complejos.

Entonces, la experiencia del taller debía posibilitar a los participantes, la construcción del andamiaje necesario para la comprensión de la metodología de trabajo interdisciplinario. Se condensaría en el proceso vivencial, el primer momento introductorio de la carrera tratando problemas del contexto jurídico y marcos disciplinares epistemológicos del Derecho, la Psicología y la Metodología de la Investigación, en función de la paulatina elaboración de un marco teórico común. Así, a través de la discusión participativa y la producción colaborativa, se propiciaría habilitar el abordaje de problemáticas específicas a tratar en los seminarios posteriores, en las prácticas interdisciplinarias tutoriadas en campo y en el horizonte temático hacia donde cada futuro/a especialista podría enfocar el trabajo final integrador.

A continuación, se referirán aspectos teóricos-metodológicos del taller "Intervenciones Interdisciplinarias" de la especialización sobre problemáticas subjetivas del contexto jurídico, fundamentados en la noción del Dispositivo Hipermedial Dinámico (DHD) aplicada a contextos educativos y de investigación (San Martín, 2008). Posteriormente se describirán aspectos relativos a lo desarrollado en el taller correspondiente a la actual cohorte y se reflexionará sobre algunos de los resultados alcanzados provisoriamente por las/los participantes en relación a las problemáticas señaladas. Finalmente las conclusiones abordarán en un orden más general, los aportes que esta experiencia podría brindar a la formación de posgrado y a las prácticas profesionales, que se orienten al desarrollo del trabajo interdisciplinario a través de redes sociotécnicas colaborativas y plurales.

\section{Fundamentos teóricos-metodológicos}

\section{Sobre la noción de Dispositivo Hipermedial Dinámico}

La pérdida de la posibilidad de todo sujeto de ser pensado y escuchado por otro, irremediablemente lo inhibe del desarrollo de un pensamiento propio y efectivo a partir del cual constituirse. En la medida en que el ajuste discursivo de la sociedad revele sólo el lazo con lo económico, la población expulsada queda fuera del lazo social, de su condición humana comprendida en la posibilidad de formar parte del universo discursivo (Lewkowicz, 2004). Si no se puede organizar ninguna respuesta, ninguna resistencia, sólo queda el acto violento, descarnado, que se estructura sobre la base de la imposibilidad de ser sujeto reconocido en su discurso, en su palabra, en su potencial creativo. 
La necesidad de construcción de un contexto físico-virtual inclusivo, instala debates sobre sus efectos en los procesos organizacionales y productivos, atendiendo a modalidades de interacción comunicacional (Yus, 2010), calidad y tipos de mediaciones y mediatizaciones, disponibilidad de infraestructura TIC y de servicios, adaptabilidad, flexibilidad y dinamismo de los sistemas digitales en uso, atención a la diversidad cultural en el contexto de implementación, a metodologías de trabajo individual y colectivo para la apropiación, producción y diseminación abierta del conocimiento a través de redes sociotécnicas colaborativas y de participación ciudadana para la calidad de vida, problemáticas que se constituyen centrales en las agendas de investigación, desarrollo e innovación de Argentina (San Martín, Guarnieri, Rodriguez, Bongiovani y Sartorio, 2010).

Las instituciones se reconfiguran a partir de este nuevo contexto físicovirtual y los cambios no dependen únicamente de disponer de los insumos tecnológicos y la conectividad a internet, sino de las apropiaciones que cada sujeto en relación con el otro pueda realizar (Thomas y Buch, 2008, García Canclini, Cruces y Urteaga, 2012). Es sabido que las tecnologías WEB integradas a contextos educativos y/o profesionales posibilitan múltiples accesos a la información, edición y modos de comunicación (procesos de circulación). Esto interroga básicamente sobre dos aspectos indisociables: el primero atiende a qué tipo de interactividad-interacción sería deseable en las diversas prácticas con distintos grados de mediatización; el segundo, plantea cuál sería la forma adecuada de llevar adelante un seguimiento de las interactividades e interacciones realizadas en contextos físico-virtuales, en función de evaluar la construcción de conocimiento y motivar la reflexión metodológica sobre la calidad de los procesos puestos en obra o posibles de desarrollar.

En atención al estudio de estas problemáticas, se definió la noción de Dispositivo Hipermedial Dinámico (DHD) como una red heterogénea, conformada por la conjunción de tecnologías y aspectos sociales -red sociotécnica- que posibilita a los sujetos en el actual contexto físico-virtual, realizar acciones participativas de interactividad -responsable- para gestionar, educar, investigar y producir bajo la modalidad de taller, utilizando la potencialidad comunicacional, transformadora y abierta de lo hipermedial (San Martín, 2008).

A partir de esta primera noción, se efectuó una estudio en profundidad de distintas perspectivas sobre los sistemas complejos (Gell-Mann, 1995, Bar-Yam, 1997, García, 2007, Morin, 1997, Reynoso, 2011, entre otros) con la finalidad de fundamentar el modelado del modo interactivo del DHD (Rodriguez, 2011), a la vez que fue efectuada una revisión histórica sobre los términos interacción e interactividad (Guarnieri, 2011).

Sobre las dimensiones que conforman al DHD, y en función de la finalidad del presente escrito, se expondrá sintéticamente a qué refiere el concepto de "Interactividad-DHD" que se constituye en un proceso clave posibilitador de la construcción interdisciplinar. 
En décadas recientes, la interactividad ha dejado de ser la primitiva y simple relación cerrada entre el individuo y la computadora aislada de toda comunicación en red -Interactividad Hombre-máquina- para ser conceptualizada a partir de nuevos escenarios relacionales y herramientas informáticas colaborativas, bajo perspectivas constructivistas y postulados que adhieren a la diseminación abierta de la información y el conocimiento (Acceso Abierto y Código Abierto). Dada esta situación de convergencia (Jenkins, 2008), si se asume un sujeto dialógico -que escucha- constructor de contenidos y artefactos, con capacidades críticas que se despliegan en el propio acto responsable, acto social instituido en la presencia de otro (Cullen, 2004), es posible que se puedan realizar un sinnúmero de comunicaciones y acciones que dan consistencia a lo humano en sus múltiples dimensiones. La pluralidad de los actos, de los actores y los artefactos, habilitaría procesos de Interactividad e Interacción en el fluir dinámico de la transformación, entretejiendo nuevas dimensiones del espacio-tiempo.

Escuchar es ingresar a la espacialidad que, al mismo tiempo, me penetra: pues ella se abre en mí tanto como en torno a mí, y desde mí tanto como hacia mí: me abre en mí tanto como afuera, y en virtud de esa doble, cuádruple o séxtuple apertura, un "sí mismo" puede tener lugar. Estar a la escucha es estar al mismo tiempo afuera y adentro, estar abierto desde afuera y desde adentro, y por consiguiente de uno a otro y de uno en otro (Nancy, 2007, p.33).

Escuchar, observar, participar y producir en contextos físicos-virtuales educativos, investigativos y/o de producción, es dar lugar a la interacción entre sujetos, a una polifonía de procesos, interacción inseparable de los diversos modos de comunicación, de reciprocidad, de afrontar el desafío de construcción de una red donde la capacidad de participar grupalmente realizando actividades sociales y/o profesionales-técnicas para el bien común (ser para los otros) se extienda más allá de la relación diádica. Es en este vínculo "social indisociable" que la noción de DHD conceptualiza la relación Interactividad-Interacción, ya que la interactividad integra cuestiones propias del actual contexto físico-virtual propuesto como red social -responsable- mediatizada por artefactos digitales. Entonces, es factible pensar los vínculos indisociables que constituyen al contexto físico-virtual, como el trazado de $n$ dimensiones complejas.

$\mathrm{N}$-dimensiones trazadas en la materialidad física del extenso campo que involucra el cuerpo, las acciones y los objetos situados en un tiempo y lugar, configurados por lo virtual en sus dimensiones de pensamiento, acto, discursos y artefactos.

Esta representación conceptual, excede la simple distinción positiva que generalmente alude a la exterioridad del ciberespacio o centra su discurso únicamente en el artefacto tecnológico. Lo propuesto sostiene que en una comunidad, las dimensiones que configuran lo virtual emergen como proceso y producto cultural portador de memorias y prácticas tanto individuales como colectivas. A partir del acceso y condiciones de intercambio dinámico de infor- 
mación, estos procesos y productos pueden ser enriquecidos, amplificados y complejizados en su propio devenir habilitándose nuevas posibilidades para la generación del conocimiento.

Desde esta perspectiva, la interactividad posibilita intercambios bidireccionales o multidireccionales siendo innegable el aporte que brindan actualmente las TIC, no obstante es necesario puntualizar que su puesta en obra tanto en campos educativos como profesionales, implica un cambio gradual de prácticas unidireccionales hacia otras donde lo bidireccional y multidireccional de lugar a una construcción participativa, abierta y plural del conocimiento. Cabe señalar, que estos procesos de cambio cultural se presentan visiblemente más lentos y trabajosos que la propia versatilidad de las tecnologías digitales (Bauman, 2009).

El descubrimiento, la exploración, la posibilidad de pensar múltiples recorridos e intercambios, habilitan nuevas prácticas donde hoy detectamos el impacto masivo de las redes sociales como por ejemplo "Facebook". Pero si bien estos sistemas muestran un desarrollo tecnológico importante, aún el trabajo colaborativo y plural, el vínculo intersubjetivo -responsable-, el acceso abierto y democrático a la información y conocimiento calificado, el respecto por la diversidad son propósitos que solicitan un compromiso activo (ético), que haga presencia ante la compleja realidad del actual contexto físico-virtual globalizado, compromiso pendiente que no registra aún el significativo número de participantes que habitan las distintas redes sociales del ciberespacio.

Entonces, habilitar procesos de "Interactividad-DHD" en contextos educativos, investigativos y lo de producción, es promover y desplegar bajo una dinámica participativa, la construcción de vínculos intersubjetivos (responsables-) mediatizados por las TIC, que construyan una red sociotécnica posibilitadora del intercambio y edición bidireccional o multidireccional de mensajes y objetos en un marco de trabajo colaborativo, abierto, democrático y plural.

De allí, que la Interactividad-DHD, solicita de la presencia del sujeto en su dimensión simbólica, más allá de la "distancia" y positividad física, haciendo foco analítico en qué es lo más adecuado según el caso para llevar adelante la tarea. Esto implica un análisis detenido sobre el perfil de requerimientos de la problemática a tratar en relación a las modalidades comunicativas, interpretativas, posibilidades tecnológicas y de producción de la comunidad interviniente, entre otros aspectos no menos importantes que solicitan un diseño físico-virtual. Entonces, lo significativo se centra en evaluar qué grado de mediatización puede resultar más conveniente para desarrollar las distintas actividades que se proponen en directa relación a las necesidades de los destinatarios. Por lo tanto, la mayor dificultad radica en sopesar alternativas, discutir y consensuar dinámicamente entre las/los participantes cómo se puede ir construyendo el Dispositivo Hipermedial Dinámico como conjunción de rasgos heterogéneos tanto sociales y como tecnológicos. Lo planteado puede ser aplicable tanto para los contextos académicos y profesionales como para los ámbitos de pro- 
ducción ciudadana y pone en foco la importancia de la elección adecuada de las tecnologías intervinientes para hacer factible y sustentable el dispositivo según el caso.

En síntesis, la noción de DHD busca promover en el actual contexto físicovirtual, procesos que habilitan y despliegan a través de la dinámica de taller, un aprendizaje hacia la participación plural, abierta, la utilización crítica de los recursos tecnológicos, entablando un diálogo investigativo amplio, no exento de dificultades, que observa y evalúa la pertinencia de las relaciones establecidas dentro y entre los subsistemas involucrados, referenciados a sus respectivas historias para finalmente interpelar en cada caso, sobre el tránsito favorable o no por alguno de los múltiples senderos posibles.

\section{Sobre el saber "ha-ser":}

Desde las últimas décadas del siglo $\mathrm{XX}$, las diferentes perspectivas y discusiones sobre la "Interdisciplinariedad" estuvieron presentes en el ámbito académico, investigativo y profesional (Follari, 2005), recobrando especial protagonismo en el campo de las Ciencias de la Educación (Cullen, 1997). En particular, las sucesivas reformas curriculares de nivel de grado y posgrado y perspectivas sobre el desarrollo de la Ciencia y la Tecnología sustentable, enfatizan con un sinnúmero de matices y posicionamientos ideológicos, la necesidad de desarrollar una formación superior centrada en el desarrollo de "competencias profesionales" que posibiliten el trabajo grupal colaborativo e interdisciplinario.

Es posible observar, que el desarrollo de la capacidad de participar grupalmente realizando actividades sociales y/o profesionales-técnicas para el bien común, es un valor sumamente significativo tanto para la dimensión política de lo educativo como de lo que constituye Subjetividad (Bauman, 2010). Sin embargo, un análisis crítico socio-histórico evidencia con innumerables hechos, la existencia de prácticas sociales fuertemente excluyentes (Lewkowicz, 2004, Esposito, 2011). Ante los múltiples escenarios de violencia, pobreza y exclusión que presenta el actual contexto físico-virtual globalizado (Monegal, Torres y Ridao, 2004), un significativo número de autores refiere la imprescindible necesidad de desarrollar capacidades que sintetizan en el sujeto el saber hacer - saber ser (saber "ha-ser") que posibiliten construir un hábitat global inclusivo y sustentable.

Si "la globalización no será soportable salvo si es pensada no como un proceso de uniformación de los pueblos y las culturas, sino como un proceso de unificación que se nutre de su diversidad en lugar de hacerla desaparecer" (Supiot, 2007, p. 292), es imprescindible sostener el esfuerzo que demanda la tarea de pensar y hacerse cargo de lo común (Cruz, 1999), generando un encuentro grupal que sin diluir la singularidad, pueda efectuar preguntas de base dando cuenta del reconocimiento general de los problemas que involucran la vida misma, para luego generar posibles soluciones (García Canclini, 2004). 
Entonces, este saber "ha-ser" más allá de la singularidad disciplinar, solicita de la construcción de un vínculo intersubjetivo (asumido como responsable), que a través de articuladas coordenadas de acción posibilite procesos participativos donde la preocupación por el bien común y la recuperación de lo público sea políticamente de todos.

En este sentido, el taller "Intervenciones Interdisciplinarias", se diseña teniendo presente que los participantes puedan vincularse a través de procesos de Interactividad-DHD que posibiliten discutir e interpretar grupalmente las complejidades sociales emergentes, indagando y reflexionando sobre los saberes disciplinares, la teoría de los sistemas complejos y las problemáticas de la interacción interdisciplinaria para la producción conjunta y colaborativa. Se interroga entonces, sobre cómo articular construcciones conceptuales y metodológicas donde lo común se sostenga aceptando la responsabilidad ética de "asumir responsabilidad por esta responsabilidad" (Bauman, 2009, p.148) ya que en la delimitación de un sistema complejo:

El análisis de cada nivel requiere de enfoques específicos que dependen de su composición, de las escalas de los fenómenos que fueron tomados en cuenta en la construcción del sistema y de las dinámicas de los procesos que tienen lugar en él. Pero el análisis del funcionamiento de la totalidad no resulta simplemente de la suma de los análisis de cada nivel. No sólo es necesario tomar en cuenta las interrelaciones entre los diferentes niveles sino también las interrelaciones conceptuales, teóricas e interpretativas (García, 2007, p.189).

A partir de los expuesto, se constituye significativo el aporte epistemológico constructivista del mencionado autor sobre el campo teórico de los sistemas complejos, referenciado a su vez en el marco teórico-metodológico del DHD, para desarrollar en el trayecto de formación de las/los futuros especialistas herramientas conceptuales y metodológicas que favorezcan múltiples y originales síntesis de acción en el tratamiento interdisciplinario de las actuales problemáticas subjetivas del contexto institucional jurídico.

Estas acciones estarían sustentadas conceptualmente en la relación Responsabilidad-Subjetividad, como posicionamiento ético, presente en el itinerario de los seminarios como desarrollo transversal, lo que posibilitaría la ineludible construcción compartida sobre la relación ciencia-sociedad que otorga consistencia al quehacer interdisciplinario para definir, bajo un mismo enfoque, el sistema complejo a intervenir (tratamiento de la problemática).

\section{La puesta en obra del Taller}

El taller "Intervenciones Interdisciplinarias", ubicado en el trayecto medio de formación, solicitó hacer tangibles los procesos de exploración, interpretación, diseño, integración y creación, tanto a nivel de propuesta curricular como en su puesta en obra. Siguiendo a Silvestri y Blank (1993), estos procesos en el marco de la acción, el pensamiento y los múltiples lenguajes expresivos, se 
pueden vincular diacrónica y sincrónicamente tanto en cada sujeto como en el grupo, a través de relaciones con jerarquías variables, singulares y dinámicas, configurando $n$ secuencias heterogéneas -polifonía de procesos- en función de las construcciones cognitivas individuales y colectivas (San Martín, 2003).

Entonces, la estrategia metodológica debía propiciar en los participantes que, tanto los contenidos como las actividades analíticas y de producción en torno a problemas observados, fueran abordados a partir de su experiencia profesional y según sus ponderaciones disciplinarias. A su vez, a nivel grupal era deseable que emerjan recorridos habilitadores de la construcción teórica común a partir de procesos de Interactividad-DHD.

Esta interacción dialéctica y abierta planteada entre el todo y las partes, caracteriza también a los sistemas complejos, dando cuenta de cómo los contenidos a tratar, se articulan en el diseño curricular con la perspectiva metodológica y concepción de formación profesional de posgrado puesta en obra.

A nivel de objetivos específicos curricularmente se propuso:

- Analizar a través de modalidades participativas físico-virtuales los conceptos de Disciplina, Multidisciplina, Interdisciplina y Transdisciplina.

- Reflexionar sobre "Interdisciplina" en el marco paradigmático de las Ciencias Sociales, reconociendo una perspectiva ética sobre los valores de la Subjetividad y los Derechos Humanos.

- Interpretar la problemática del contexto jurídico-forense, desarrollando un enfoque constructivista sobre los sistemas complejos.

- Diseñar instrumentos que favorezcan el trabajo interdisciplinario en función de intervenciones efectivas.

- Producir informes técnicos elaborados bajo un marco teórico común de carácter interdisciplinario.

En continuidad con las temáticas y bibliografía tratada en seminarios anteriores, el taller se diseñó a partir de los siguientes tres módulos de contenidos: Módulo I: Concepto de Disciplina, Multidisciplina, Interdisciplina, Transdisciplina - Perspectiva epistemológica de la Interdisciplina - El impacto de las tecnologías de la Información y Comunicación. La ética con la Subjetividad y los Derechos Humanos.

Módulo II: Sistemas complejos e interdisciplinariedad: caso el contexto jurídico - El diálogo y la participación responsable como estrategia de cambio organizacional - La problemática de distancia disciplinar: prejuicio y preconcepto - Estrategias para la construcción del marco teórico-metodológico común. La conformación de equipos de investigación: instrumentos y técnicas para lo interdisciplinario.

Módulo III: El informe técnico: producción interdisciplinar - Hacia una lectura y gestión transversal: Diagnóstico, pronóstico, orientación y tratamiento. La participación en equipos de gestión y asesoramiento en prevención y promoción en el ámbito de la Políticas públicas. 
Cabe aclarar que las/los estudiantes acreditaban conocimientos sobre enfoques y perspectivas de la complejidad, especialmente lo desarrollado por Edgar Morin, sin embargo desconocían el marco teórico y metodológico propuesto por Rolando García y el modelo de mediatización físico-virtual implementado a partir de la noción del DHD.

En cuanto a los tiempos de cursado del taller físico-virtual, se realizaron en la universidad 3 jornadas consecutivas de trabajo (18 horas reloj) y luego se continuó trabajando en el espacio virtual realizando diversas actividades colaborativas durante 4 meses (sin incluir el mes de receso). El taller acreditaba un total de 30 hs. reloj y el espacio virtual del taller diseñado ad hoc, utilizó como entorno colaborativo Moodle.

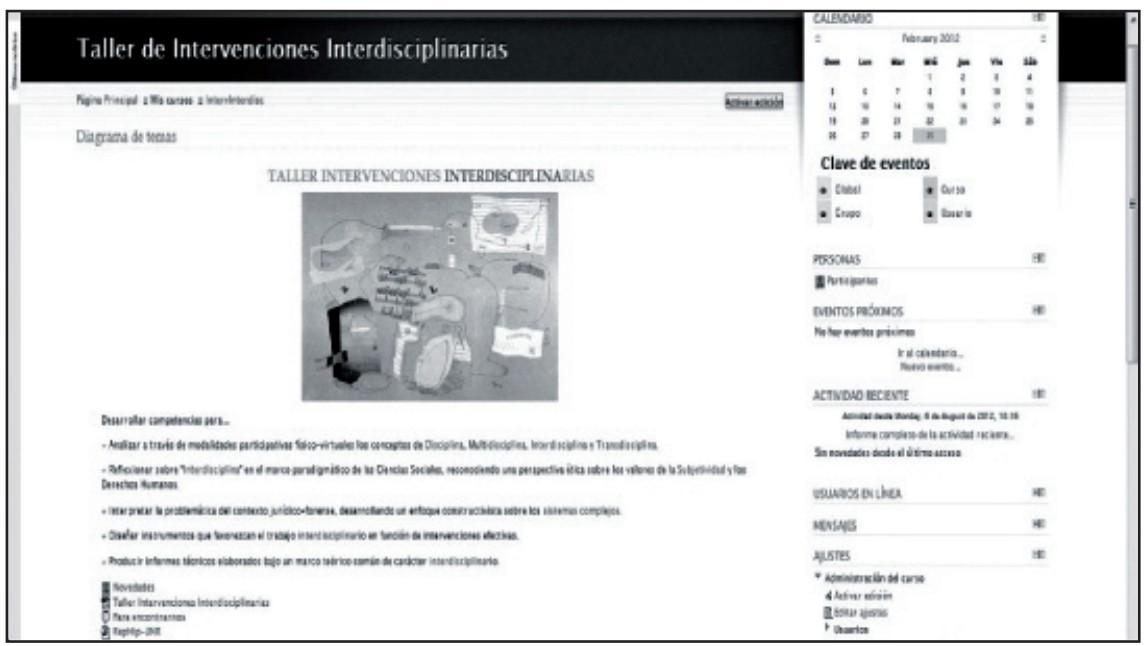

Figura 1: Fragmento del taller en Moodle

Para posibilitar una mejor comprensión de lo propuesto, se ha habilitado el ingreso abierto a dicho entorno (http://dimensionesdhd.cifasis-conicet.gov. ar/course/view.php?id=29) bajo el rol de "invitado" donde se accede en parte a los procesos de Interactividad-DHD. Cabe aclarar que por razones académicas no se muestran la totalidad de los materiales, herramientas utilizadas, producciones y evaluaciones realizadas.

Sobre el grupo de cursantes, los mismos provenían profesionalmente del campo de la Psicología, Trabajo Social, Derecho y Educación, la mayoría acreditaba diversas experiencias laborales en el contexto jurídico en relación a problemáticas de violencia familiar, educación en contextos de encierro, menores en riesgo social, abuso infantil, entre otras. Las/os participantes poseían una alfabetización digital básica, correo electrónico, acceso a redes sociales y 
técnicas de búsqueda de información, además el $25 \%$ había realizado algún curso virtual utilizando Moodle. Sobre un total de 26 estudiantes, se registraron 5 ausentes y 21 cursantes activos.

Para la acreditación del taller se debía cumplimentar un $80 \%$ de presencia física-virtual. Lo cual se efectivizó luego de las 3 jornadas en la universidad, a través de distintas actividades consecutivas en el espacio virtual del taller.

El diseño del taller concibió desde un principio el espacio físico-virtual: las/ los asistentes contaban con sus computadoras portátiles y acceso al entorno virtual una semana antes del inicio de las jornadas en la universidad (momento de inicio de las actividades). En el aula de la institución, se fueron colocando los pupitres móviles de acuerdo a las necesidades grupales y se utilizó simultáneamente el pizarrón, proyector multimedia, equipo de sonido, pantalla, material de biblioteca, notebooks y acceso inalámbrico a Internet brindado por la universidad.

Las condiciones tecnológicas aparentemente no presentaban dificultades, sin embargo la conectividad simultánea de más de 15 computadoras portátiles efectuando sincrónicamente diversos procesos de edición y comunicación en línea, bajó significativamente la velocidad de transferencia de datos, aspecto que da cuenta de las debilidades que aún se registran en un número significativo de localidades de Argentina con referencia a capacidades de conectividad, acceso y estabilidad de la conexión a Internet en el marco de requerimientos de edición colaborativa y visualizaciones con formato de video.

La dinámica del cursado físico-virtual contempló que en los encuentros en la universidad se tratara el desarrollo de los contenidos curriculares integrando cuestiones operativas del entorno virtual, con la finalidad de asegurar la accesibilidad y la realización de las actividades de discusión, lectura y edición bajo el concepto de Interactividad-DHD.

Así, lo tratado y debatido en los encuentros en la universidad, se siguió elaborando a través de los foros, chat y otros recursos de estudio (artículos, presentaciones, videos, etcétera) configurados en el espacio virtual y coordinados por la responsable del taller. A la vez, cabe mencionar que el grupo sostenía encuentros auto-regulados en la institución. Huellas de esta dinámica, quedaron registradas en las conversaciones por chat del entorno virtual.

Como ejemplos de las actividades de aprendizaje efectuadas, es posible mencionar:

*Búsqueda de información ampliatoria de los temas tratados en los módulos: se habilitó una wiki donde las/los cursantes fueron consignando un directorio de enlaces en internet a instituciones, centros de investigación, repositorios institucionales de acceso abierto, diversas organizaciones, autores de referencia, publicaciones de interés en distintas áreas disciplinares a nivel regional, nacional e internacional, efectuando sintéticos comentarios. También 
se planteó un foro de interconsulta sobre bibliografía ampliatoria en función de necesidades específicas de los trabajos grupales.

*Participación en debates físicos-virtuales: el taller se inicia con un foro físico-virtual donde cada participante presenta su narrativa individual sobre experiencias de trabajo profesional grupal, reflexionando acerca de la misma como buena práctica o no de trabajo interdisciplinario.

${ }^{*}$ Análisis individuales y conjuntos sobre problemáticas del trabajo interdisciplinar en las instituciones: siguiendo lo anterior, cada participante indexó en forma individual lo que interpretaba como obturadores al despliegue de un trabajo interdisciplinario en distintos ámbitos institucionales. El envío del trabajo lo realizaron utilizando la herramienta "tareas" de Moodle.

*Edición colaborativa de textos con diversas funcionalidades en formato wiki: en este sentido se trabajó metodológicamente sobre la construcción textual colaborativa en función de la construcción del marco teórico común. La primera actividad correspondiente al segundo módulo, se centró en la construcción de un texto de autoría conjunta (consensuado por la totalidad de talleristas) que expusiera las problemáticas que obturaban el trabajo interdisciplinario en el contexto jurídico. El mismo debía contener una introducción, descripción y análisis teórico de las problemáticas desde los marcos epistemológicos abordados y conclusiones prospectivas. Como parte del proceso se efectivizó la construcción simultánea de un "glosario" disciplinar de términos, propuesto como una estrategia para consensuar grupalmente la perspectiva epistemológica de las terminologías puestas en juego.

La segunda actividad que se desarrolló en la última fase, propuso a las/los participantes que formen grupos a partir de la libre elección e intereses y desarrollen qué problemática de estudio en conjunto deseaban abordar, exponiendo la delimitación de dicho sistema complejo y la temática específica disciplinar que estudiaría cada uno de los integrantes. Se recomendó tener en cuenta que la actividad era conducente hacia el trabajo final de la especialización donde se contemplaba un taller de desarrollo y la guía a cargo de un tutor específico.

Cada actividad contempló precisas instrucciones destinadas a las/los talleristas con la finalidad de facilitar la organización de sus tareas en vistas a la acreditación del taller. Las actividades se desarrollaron de acuerdo a un cronograma que presentaba superposiciones y cada módulo fue planteado como un conjunto abierto de actividades relacionadas donde algunas de ellas solicitaban una permanente revisión, ampliación y producción -individual y grupal- de contenido.

\section{Acerca de la evaluación de la Interactividad-DHD}

Los principales inconvenientes técnicos durante se registraron por problemas de conectividad y velocidad del servicio de Internet, problemas 
informados en su mayoría por aquellas/os talleristas que habitan en pequeñas localidades del NOA. La solución de estos problemas, externos en su mayoría a la institución universitaria, aún está en vías de desarrollo en una gran parte del territorio argentino. Específicamente, se constataron inconvenientes en la fluida transmisión de datos y comunicación sincrónica y asincrónica a través de las herramientas disponibles en el entorno Moodle, por lo cual el grupo articuló bajo el concepto DHD algunos encuentros presenciales y otras comunicaciones utilizando servicios de telefonía móvil. En lo general, no se registraron problemas ni dificultades en la operatividad de Moodle.

Sobre las actividades de seguimiento y evaluación llevadas adelante por la docente coordinadora del taller, es importante destacar que los procesos de Interactividad-DHD que se desarrollaron se observaron tanto en relación al nivel que lograron las/los talleristas en la producción académica grupal como en la individual constituyéndose actualmente dicha dinámica relacional un objeto de estudio relevante. Una primera evaluación sobre las participaciones, se realizó utilizando la herramienta extendida "Informe de Actividad" que brinda la nueva versión de Moodle (2.1.2), sin embargo a la hora de realizar ponderaciones más complejas y análisis de los comportamientos de la red social del taller, las misma resulta insuficiente. Esto se debe, a que dicha herramienta no brinda la posibilidad de visualizar y ponderar cualitativamente las relaciones complejas que va generando la participación en red a través de las distintas herramientas y servicios. En este sentido, se cuenta con un primer prototipo de herramienta de código abierto desarrollada ad hoc denominada "SEPI-DHD", Seguimiento y Evaluación de Procesos de Interactividad-DHD (http://dimensionesdhd. cifasis-conicet.gov.ar:8080/SEPI-DHD/) que brinda un análisis cuali-cuantitativo de las participaciones, teniendo en cuenta roles, herramientas, servicios y configuración del Dispositivo Hipermedial Dinámico (Rodriguez y San Martín, 2010). Es importante señalar que SEPI-DHD, es una tecnología que colabora con la evaluación de los procesos de Interactividad-DHD en este caso aplicado al campo de formación profesional, pero justamente demanda al docente una reflexión profunda sobre lo que se pretende ponderar en la evaluación de dichos procesos, por lo cual ofrece una configuración abierta como instrumento evaluativo y en sus prestaciones no pretende cubrir la totalidad de los aspectos a evaluar que el diseño curricular concibe. A partir de la utilización de SEPI-DHD, se está llevando adelante un análisis evaluativo detallado de la experiencia descripta que excede los límites del presente trabajo.

Sobre la evaluación provisoria de los procesos de Interactividad-DHD, cabe destacar que las participaciones fueron configurando una dinámica polifónica y espiralada, generándose $n$ secuencias múltiples que relacionaban los distintos momentos planteados en los módulos, este aspecto se considera de máxima importancia ya que confirma una intencionalidad de la propuesta a nivel de su diseño. Por ejemplo, el foro del módulo I referido a la experiencia de trabajo 
interdisciplinar siguió sumando intervenciones que continuaban reflexionando sobre las experiencias vividas, el glosario del módulo II se incrementó en el tiempo en la medida que las/los participantes profundizaban en cuestiones de contenido, como así también los sitios de referencia de la introducción se multiplicaron para poder acceder a publicaciones e información de interés en Acceso Abierto. A su vez, la participación en debates físicos-virtuales generó expectativas e intercambios muy intensos y emotivos entre los cursantes que habilitaron nuevas producciones individuales y grupales analizando los propios textos de las intervenciones, dando cuenta de la calidad del vínculo intersubjetivo responsable que se iba gestando.

Sobre la calidad de los análisis individuales y conjuntos realizados sobre las problemáticas del trabajo interdisciplinar en las instituciones, los mismos fueron un resultado de diálogo fluido no exento de tensiones entre lo individual y grupal. Un esfuerzo importante por parte de los participantes, fue el logro de categorizaciones para establecer los distintos componentes del sistema y subsistemas del contexto jurídico. Este trabajo se fue desarrollando durante un período prolongado a través de lecturas, intercambios y producción colaborativa habilitando paulatinamente la emergencia del marco teórico común.

El proceso más complejo fue llevar adelante en formato wiki la construcción del texto de autoría conjunta donde se expusieron las problemáticas que obturaban el trabajo interdisciplinario en el contexto jurídico. Si bien esto se configuró como una primera experiencia para las/los participantes (muy lejana a sus prácticas profesionales), se logró luego de varias versiones y consultas formalizar un texto consensuado que en su propia enunciación se constituyó en una herramienta de reflexión transversal para los trabajos posteriores. La construcción simultánea del glosario de términos fue clave para consensuar la perspectiva epistemológica, contando al fin del taller con más de 50 términos, comentarios respectivos, visitas y optimizaciones frecuentes.

En la wiki desarrollada en la última fase, los grupos se mostraron más consolidados en sus intereses y se logró un nivel de coherencia aceptable en la construcción del problema, articulado desde una mirada interdisciplinar a través de los aportes de cada uno en su especificidad disciplinar. En esta dirección, lo planteado en la consigna de trabajo fue una estrategia integradora de carácter teórico, metodológico y procedimental hacia lo interdisciplinario fundamentada en el propio hacer del grupo, que solicitó como fase previa el análisis y procesamiento de información sobre casos en forma colaborativa y multidisciplinaria para arribar posteriormente a la producción del prediseño del trabajo final integrador construido en la simultaneidad individual y colectiva.

Sobre los prediseños presentados, es posible constatar diversas aproximaciones temáticas, factibilidad, posibilidades de acceso al trabajo de campo y a las fuentes bibliográficas dando cuenta de la perspectiva teórica y metodológica propuesta. 
En un orden general, los niveles de desempeño individuales y grupales de las/los participantes del taller que se pueden observar en los textos disponibles fueron heterogéneos, si bien 21 estudiantes aprobaron el taller, no todos los grupos tuvieron similares niveles en cuanto a sus aportes, conceptualizaciones e intervenciones. Sin embargo, se puede verificar que en el transcurso de la experiencia, se fue transformando el discurso voluntarista o incluso a veces desalentador que prevalecía entre los presentes sobre sus experiencias de trabajo interdisciplinario, hacia otro discurso más formalizado, reflexivo y sistemático en cuanto a enfoques e instrumentos para abordar las problemáticas planteadas y elaborar un marco teórico común. En este sentido, la revisión y discusión sobre casos fue un camino conducente hacia la reflexión sistemática grupal e individual. Retomando una de las problemáticas enunciadas en la introducción de este trabajo sobre la parcelación de las intervenciones en la práctica profesional del contexto jurídico, a continuación se cita un extracto de una wiki final, que ejemplifica sintéticamente como uno de los grupos fue elaborando un discurso analítico pertinente al marco sistémico complejo:

A modo de conclusión: Abordaje Interdisciplinario del conflicto.

En el análisis del caso judicial mencionado, advertimos que el sistema judicial realizó un abordaje segmentado de la problemática, prescindiendo de la institución familiar como sistema complejo. Cada organismo contempló el conflicto desde su órbita exclusivamente disciplinar, pasando casi inadvertida la naturaleza de la problemática de fondo, la violencia familiar, de la cual el abuso denunciado y detonante de la intervención judicial suele ser sólo un episodio...

\section{Conclusiones}

A través de este escrito, fueron presentados los fundamentos de diseño del taller "Intervenciones Interdisciplinarias", como un camino posible para el desarrollo de competencias profesionales en relación a las problemáticas subjetivas del contexto jurídico. El caso expuesto, pretende ser un aporte hacia la formación profesional de posgrado, que más allá de las acreditaciones formales, será finalmente evaluada en sus efectos por los propios actores, en el marco de sus diversas prácticas laborales.

En un orden más general, la posibilidad de acceso abierto a gran parte de lo efectuado en el entorno virtual utilizado por el taller, se configura en un antecedente documentado que posibilita seguir discutiendo el diseño e implementación de diversos talleres físico-virtuales de posgrado que aborden contenidos sobre sistemas complejos y metodología de trabajo interdisciplinario para el tratamiento de problemáticas de alta significatividad social. En esta dirección, se enfatiza la necesidad de construcción social y tecnológica de redes sociotécnicas de formación y práctica profesional, fundamentadas en la noción de Dispositivo Hipermedial Dinámico, comprometidas con la ética de la Subjetividad-Responsabilidad, los Derechos Humanos y la consecuente 
adhesión a las perspectivas internacionales de Acceso Abierto (Educación, Conocimiento Científico y Tecnológico) y Código Abierto.

Cabe afirmar que, el taller "Intervenciones Interdisciplinarias" concebido como caso propositivo contextualizado, motiva a reflexionar sobre cómo los aportes del epistemólogo Rolando García sobre los sistemas complejos, sustentan el concepto de "Interactividad-DHD", habilitando la puesta en obra de una dinámica de producción interdisciplinaria a través de una polifonía de procesos y secuencias múltiples, en el marco de la acción, pensamiento y lenguajes.

Por último, la experiencia narrada presenta un proceso de aprendizaje significativo hacia el saber "ha-ser" donde subyace una dialéctica constructiva que tensa y entrama las $n$ dimensiones de lo físico y lo virtual, a partir de trayectos en construcción intersubjetiva. Así, el permanente devenir entre lo singular y lo colectivo, la presencia y la ausencia, lo disciplinar y lo interdisciplinar, lo sincrónico y lo asincrónico, estará siempre atravesado por el desafío constante de "cómo iluminar la elección para sostener la decisión" (Badiou, 2005, p.16).

\section{Referencias bibliográficas}

- $\quad$ Badiou, A. (2005). Filosofía del presente (10 Ed.). Buenos Aires: Libros del Zorzal.

- Bauman, Z. (2010). Mundo consumo. Ética del individuo en la aldea global (1 Ed.). Buenos Aires: Paidós.

- $\quad$ Bauman, Z. (2009). El arte de la vida. De la vida como obra de arte (1º Ed.). Buenos Aires: Paidós.

- Bar-Yam, Y. (1997). Dynamics of complex systems (1 Ed.). New England: AddisonWesley.

- $\quad$ Cruz, M. (1999). Hacerse cargo. Sobre responsabilidad e identidad personal (1 Ed.). Barcelona: Paidós.

- Cullen, C. (1997). Crítica de las razones de educar. Temas de filosofía de la educación $\left(1^{\circ} \mathrm{Ed}\right.$.). Buenos Aires: Paidós.

- Cullen, C. (Compilador) (2004). Filosofía, cultura y racionalidad crítica. Nuevos caminos para pensar la educación (1 ${ }^{a}$ ed.). Buenos Aires: Stella, La Crujía.

- Degano, J. (2005). La ficción de la rehabilitación, Prácticas judiciales actuales y políticas de la subjetividad ( $1^{\circ} \mathrm{Ed}$.). Rosario: Juris.

- Degenne, A. (2009). Tipos de interacciones, formas de confianza y relaciones. REDES, Revista hispánica para el análisis de redes sociales, 16(3), 63-91.

- $\quad$ Esposito, R (2011). El dispositivo persona (1 Ed.). Buenos Aires: Amorrortu.

- $\quad$ Follari, R. (2005). La interdisciplina revisitada. Andamios, 1(2), 7-17.

- García Canclini, N. (Coordinador) (2004). Reabrir espacios públicos. Políticas culturales y ciudadanía (1 Ed.). México: Universidad Autónoma Metropolitana Unidad Iztapalapa. Plaza y Valdés, S.A de C.V.

- García Canclini, N, Cruces, F. y Urteaga Castro Pozo, M. (Coordinadores) (2012). Jóvenes, Culturas Urbanas y Redes Digitales ( $1^{\circ} \mathrm{Ed}$.). Buenos Aires: Ariel.

- García, R. (2007). Sistemas Complejos. Conceptos, método y fundamentación epistemológica de la investigación interdisciplinaria ( $1^{\circ} \mathrm{Ed}$.). Buenos Aires: Gedisa.

- $\quad$ Gell-Mann, M. (1995). El quark y el jaguar. Aventuras en lo simple y lo complejo (1 Ed.). Barcelona: Tusquets. 
- $\quad$ Guarnieri, G. (2011). El modo interactivo del Dispositivo Hipermedial Dinámico (1 Ed.). Rosario: Universidad Nacional de Rosario.

- Jenkins, H. (2008). Convergence Culture. La cultura de la convergencia de los medios de comunicación (1ºd.). España: Paidós.

- $\quad$ Lewkowicz, I. (2004). Pensar sin estado. La subjetividad en la era de la fluidez ( $1^{\circ} \mathrm{Ed}$.). Buenos Aires: Paidós.

- Monegal, A., Torres, F. y Ridao, J. M. (compiladores) (2004). En guerra (10 Ed.). Barcelona: Centre de Cultura Contemporánia de Barcelona, Institut d’Edicions, Diputació de Barcelona.

- Morin, E. (1998). Introducción al pensamiento complejo (2 Ed.). Barcelona: Editorial Gedisa.

- $\quad$ Nancy, J. L. (2007). A la escucha (1 Ed.). Buenos Aires: Amorrortu.

- Reynoso, C. (2011). Redes sociales y complejidad: Modelos interdisciplinarios en la gestión sostenible de la sociedad y la cultura ( $1^{\circ} \mathrm{Ed}$.). Ciudad Autónoma Buenos Aires: Editorial Sb.

- Rodríguez, G. (2011). La teoría de los sistemas complejos aplicada al modelado del Dispositivo Hipermedial Dinámico ( $1^{\circ}$ Ed.). Rosario: Universidad Nacional de Rosario.

- Rodríguez, G., San Martín P. (2010). Approximation to the Dynamic Hypermedial Device modeling for the analysis of interactions. Journal of Computer Science and Technology, 10 (3) 123-129.

- $\quad$ San Martín, P. (2003). Hipertexto: Seis propuestas para este milenio (1º Ed.). Buenos Aires: La Crujía.

- San Martín, P., Guarnieri, G., Rodríguez, G., Bongiovani, P. y Sartorio, A. (2010). El Dispositivo Hipermedial Dinámico Campus Virtual UNR (1 Ed.). Rosario: Universidad Nacional de Rosario.

- San Martín, P. (2008). Hacia la construcción de un Dispositivo Hipermedial Dinámico. Educación e investigación para el campo audiovisual interactivo ( $1^{\circ} \mathrm{Ed}$.). Buenos Aires: Universidad Nacional de Quilmes Editorial.

- $\quad$ Silvestri A. y Blank G. (1993). Bajtin y Vigotsky: la organización semiótica de la conciencia (1 Ed.). Barcelona: Anthropos.

- Supiot, A. (2007). Homo Juridicus. Ensayo sobre la función antropológica del derecho ( $1^{\circ}$ Ed.). Buenos Aires: Siglo XXI Editores.

- $\quad$ Thomas, H.y Buch, A. (compiladores) (2008). Actos, actores y artefactos ( $1^{\circ} \mathrm{Ed}$.). Buenos Aires: Universidad Nacional de Quilmes Editorial.

- Yus, F. (2010). Ciberpragmática 2.0. Nuevos usos del lenguaje en Internet (10 Ed.). España: Ariel. 